\title{
Knowledge creation: synthesis, tools and products
}

\author{
Melissa Brouwers PhD, Dawn Stacey RN PhD, Annette O'Connor RN PhD
}

$\mathrm{T}$ he foundation for any activity related to knowledge translation is knowledge; it's hard to do KT without the "K." In the first article of this series, we introduced the knowledge-to-action cycle, which provides a framework for strategies in knowledge translation. In the centre of this cycle is the knowledge funnel, which represents knowledge creation. ${ }^{1}$ As knowledge moves through the funnel, it is refined and, ideally, becomes more useful to end-users of the knowledge. These end-users can include clinicians, researchers, policy-makers and the public. During each phase of knowledge creation, producers of knowledge tailor their activities to the needs of these end-users.

First-generation knowledge is that which is derived from primary studies such as randomized trials. Knowledge synthesis represents second-generation knowledge; systematic reviews are examples of this knowledge-related product. Third-generation knowledge includes tools and products such as decision aids and educational modules whose purpose is to present knowledge in user-friendly, implementable formats.

Implementation of evidence from individual studies may be misleading because of bias in the conduct of studies or random variations in findings. This fact has led to greater emphasis on knowledge syntheses as the foundation of efforts to implement knowledge. ${ }^{2}$ Knowledge syntheses serve to interpret the results of individual studies within the context of all of the evidence. These syntheses provide the evidence base for other tools of knowledge translation, such as decision aids for patients, clinical practice guidelines or policy briefs. For further discussion of the types of knowledge syntheses available, see a recent article by Ioannidis that describes them more fully. ${ }^{3}$ We need to avoid the "knowledge translation imperative," or the assumption that the results of every study should be implemented. Instead, we should focus on areas where a mature and valid evidence base exists.

Knowledge tools such as guidelines for clinical practice and decision aids for patients can be used by clinicians and patients to make evidence-based decisions. The goals of using these tools are to provide an explicit, evidence-based description of the risks and benefits of an intervention and to outline areas where evidence is lacking. Many situations exist where sufficient evidence is lacking or the balance between risks and benefits is nearly even ${ }^{4}$ and this highlights the importance of clinical expertise and exploration of the values and circumstances of patients.

\section{Key points}

- Systematic reviews form the foundation of many activities related to knowledge translation.

- High-quality clinical practice guidelines and decision aids can improve the quality of decisions and care.

- Ongoing efforts to improve the application of clinical practice guidelines and decision aids are warranted.

\section{What are clinical practice guidelines?}

Evidence-based guidelines for clinical practice are knowledge tools that are systematically developed statements aimed at assisting clinicians and patients to make decisions about appropriate health care for specific clinical circumstances. ${ }^{5}$ Clinicians are bombarded with practice guidelines, as seen in guideline databases, ${ }^{6}$ which contain thousands of these tools. Practice guidelines may also inform decisions made by health care policy-makers and clinical managers. ${ }^{7}$ In addition to many other factors (e.g., the values and preferences of patients, care providers and society) guidelines are intended to assist decision-making but not supplant it. They are not dictum-like or formulaic tactics to drive patient care. Guidelines should state whose viewpoint was considered in the development of the guidelines and provide a framework for clinicians and patients to use when deciding how to implement the guidelines.

\section{How are practice guidelines developed?}

Systematic and rigorous methods exist to ensure that highquality clinical practice guidelines are created. ${ }^{8-13}$ The development of guidelines requires a combination of social engagement and methodological rigour (Box 1). Ensuring that the appropriate stakeholders are involved is a key strategy to facilitate successful development and implementation of guidelines. Stakeholders may include clinicians, researchers, funders, policy-makers, patients and others. Ideally, the group carrying out the development should be composed of a multidisciplinary team of stakeholders, including clinical and content-specific experts, methodologists and other end-users, including patient representatives. ${ }^{14}$

From the Department of Oncology and the Department of Clinical Epidemiology and Biostatistics (Brouwers), McMaster University, Hamilton, Ont.; the School of Nursing (Stacey, O'Connor), University of Ottawa, and the Ottawa Hospital Research Institute (Stacey, O'Connor), Ottawa, Ont.

Cite as CMAJ 2009. DOI:10.1503/cmaj.081230 
Box 1: Steps in the development of clinical practice guidelines

- Establish multidisciplinary guideline team.

- Identify clinical question that explicitly defines the patients, intervention or exposure, comparisons (if relevant), outcomes of interest and setting.

- Conduct a systematic review of evidence.

- Appraise and interpret evidence and come to consensus on its meaning.

- Draft guideline recommendations that align with evidentiary base.

- Complete an external review of draft report among intended users and key stakeholders.

- Revise the guideline in response to external review.

- Prepare the final guideline report for distribution and dissemination

- Prepare strategy for implementation.

The external review of guidelines is also a form of social engagement. The process of external review can create accountability between developers and users of the guidelines, and provides a forum through which stakeholders can be engaged in the endorsement and implementation of the guidelines. It is only with the engagement of relevant stakeholders that a viable strategy for implementation of the guidelines can be developed. ${ }^{8,9}$

Methodologically, clinical practice guidelines begin with a clinical question informed by a clinical or care-related problem. The PICO (Patient, Intervention, Comparison, Outcome) format can be used to formulate the question. This question informs the inclusion and exclusion criteria that will be used to complete a systematic review of the evidence. Ideally, guidelines should include all relevant strategies (e.g., strategies for screening, diagnosis, and treatment or prognosis) and the full range of outcomes (both good and bad) that are important.

Valid guidelines create their evidence-related components from systematic reviews of relevant, worldwide literature. ${ }^{15}$ This evidence base serves as the foundation for developers to make clinical recommendations. The reviews are "necessity"-driven and synthesize the best evidence (even if it's of shaky quality) that can be found to guide a decision. Thus, some recommendations in a guideline may be derived from high-quality evidence and others from evidence that is much more liable to error. ${ }^{15}$ Given that the quality of evidence may vary, it is useful to have the strength of the recommendations graded on the quality of evidence found. Although variability exists in the methodological approaches to this grading, developers and users of guidelines agree that a need exists for a clear link between recommendations and the evidence that supports them. ${ }^{16,17}$

The GRADE (Grading of Recommendations Assessment Development and Evaluation) method is an emerging strategy favoured by those who seek a structured, categorical framework for grading the level of evidence and strength of recommendations. ${ }^{18,19}$ A clear articulation of how the developers interpreted the evidence and reached their conclusions is important. In addition to an evidence-specific component, guidelines should have a detailed instructional component about what to consider in individual patients. Although much of this instructional component needs to be individualized to local settings and patients, general frameworks can be provided that consider local variation in prevalence, feasibility, potential barriers and other variables.

The next step in developing a guideline is an external review by key stakeholders and intended users of a draft version of clinical practice guidelines. ${ }^{8-11}$ This review can improve the quality of guidelines by identifying evidence that was missed. It can also enable stakeholders to endorse the interpretation of the evidence by the developers or offer alternative interpretations. Furthermore, this review provides an opportunity to explore the "implementability" of recommendations, including barriers and enablers to their application.

Although several resources are available to guide in the development of original clinical practice guidelines, ${ }^{8-11,13}$ recent efforts by a group of international researchers known as Adapte have resulted in a rigorous methodology to enable clinical practice guidelines from one jurisdiction or context to be adapted for use in another. ${ }^{20}$ The process of adapting guidelines is discussed in a later article in this series. ${ }^{21}$ Adaptation is identified as an important step toward reducing duplication in the development of guidelines, which can result in a tremendous cost in financial and human resources.

\section{Do clinical practice guidelines work?}

The impact of clinical practice guidelines on practice and outcomes is complex. Systematic reviews by Grimshaw and oth$\mathrm{ers}^{22,23}$ suggest that interventions to implement clinical practice guidelines do, on average, influence both the processes and the outcomes of care, although the effect sizes tend to be modest at around $10 \%$. Interventions for implementation ranged from mass-media interventions to use of local opinion-leaders and include interventions targeted toward the public, health care professionals, managers and others. ${ }^{23}$ However, the potential benefits of clinical practice guidelines are only as good as the quality of the guidelines themselves. Interestingly, Grimshaw and colleagues ${ }^{23}$ found in their review of 235 studies that only 3 guidelines were explicitly evidence-based.

Although faithfulness to evidence-based principles is important, other nonscientific factors influence the uptake of guidelines. These factors include the perceptions by the adopters of the guidelines' characteristics and messages and of the development process, and factors related to norms and context. ${ }^{24}$ The likelihood of success is increased by a strategy for implementation that includes analysis of enabling factors and barriers, selection of appropriate and feasible interventions related to knowledge translation, and indicators to measure impact. These issues are addressed in later articles in this series. ${ }^{18,25-29}$

\section{How do we determine the quality of clinical practice guidelines?}

Not all clinical practice guidelines are created equal. The quality of these tools can be extremely variable and often falls short of basic standards. For example, when Graham and 
colleagues $^{27}$ appraised the quality of 217 Canadian clinical practice guidelines for drug therapy that were produced between 1994 and 1999, they found that less than $15 \%$ of those reviewed met $50 \%$ or more of the 20 criteria assessing the rigour of development. The mean proportion of criteria that were met was $30 \%$. Before efforts at implementation are undertaken, it is critical that a high-quality guideline be developed or adapted. Not unusually, different professional organizations may provide different recommendations based on the same evidence. ${ }^{30}$ These differences can result from differences in methods (e.g., search criteria, the way evidence is appraised and the way recommendations are created), in the interpretation of evidence and in health care settings.

In response to this challenge, the AGREE (Appraisal of Guidelines Research and Evaluation) Collaboration was established to develop a valid and reliable instrument for assessing the quality of guidelines (Box 2). The AGREE Collaboration defines quality of guidelines as the level of confidence that can be placed in the minimization of biases linked to the rigour of development, presentation and applicability of a clinical practice guideline and in the clear reporting of each step of the development process. ${ }^{12,13}$ The AGREE instrument is composed of 23 items answered using a fourpoint Likert scale (i.e., ranging from "strongly disagree" to "strongly agree"), one item for global endorsement answered with a four-point categorical scale, and a User Guide..$^{12,13}$ The AGREE Next Steps initiative builds on this work to improve the reliability and establish the validity of the instrument.

The AGREE instrument provides important criteria upon which to evaluate clinical practice guidelines. But the clinical validity, appropriateness of recommendations and thorough analysis of the capacity to implement recommendations are not within its scope. Indeed, although no gold standards for these measures of quality currently exist, some advances have been made. For example, the GLIA (Guideline Implementability Appraisal) tool provides a unique perspective by assessing the extent to which recommendations are implementable (Box 3). ${ }^{28,29}$

\section{What are decision aids for patients?}

Decision aids for patients translate evidence into patientfriendly tools to inform patients on their options, help them clarify the value they place on benefits versus harms, and guide them in the process of decision-making. ${ }^{31}$ As mentioned earlier, knowledge syntheses are the foundation for

Box 2: Instrument domains of the AGREE (Appraisal of Guidelines Research and Evaluation) Collaboration

- Scope and purpose of the guideline

- Involvement of stakeholders in development of the guideline

- Rigour of the process of development

- Clarity and presentation of the summary of evidence and recommendations

- Applicability of the guideline

- Editorial independence these knowledge tools. Specifically, evidence included in decision aids for patients is defined as up-to-date scientific information on options, benefits and risks of options, and associated probabilities. ${ }^{32}$ Formats for these tools include paper-based booklets, video/DVDs, decision boards and Internet-based materials. Decision aids for patients are used as adjuncts to counselling by practitioners for decisions for which the best choice depends on how patients weigh the benefits, risks and scientific uncertainty (e.g., birth control, genetic testing, breast and prostate cancer treatment, options for menopause symptoms, back pain, osteoarthritis or level of care at end of life). These decision aids differ from educational materials by not only providing information on options but also tailoring it to patients' clinical risk profiles and guiding patients to define and express their personal values. Items from a sample page of a patient decision aid are provided in Appendix 1, available online at www.cmaj.ca/cgi/content /full/cmaj.081230/DC1. Others are available elsewhere online at www.ohri.ca/decisionaid.

\section{How are decision aids developed?}

High-quality decision aids for patients are developed using a systematic process. We briefly summarize the methods of development here. Explicit guides to the process are available elsewhere. ${ }^{32}$ The first step is to determine the decision-making needs of potential users (e.g., patients and clinicians). Needs assessments focus on the users' perceptions of the decision (i.e., options, outcomes, values), perceptions of others involved in the decision (i.e., decisional roles, opinions, pressures) and resources needed to make or implement the decision. ${ }^{33}$ Second, the patient decision aid is based on a synthesis of the evidence and includes essential elements (Box 4). To

Box 3: Elements of the GLIA (Guideline Implementability Appraisal) tool for assessing guidelines ${ }^{29}$

- Decidability: precisely under what circumstances to do something

- Executability: exactly what to do under the defined circumstances

- Effect on process of care: the degree of impact of the recommendation on the usual workforce

- Presentation and formatting: the degree to which the recommendation is recognizable and succinct

- Measureable outcomes: the degree to which the guidelines identify markers or end-points for tracking the effect of implementation of the recommendation

- Validity: the degree to which the recommendation reflects the intent of the developer and the strength of the evidence

- Novelty and innovation: the degree to which the recommendation proposes behaviour considered unconventional by clinicians or patients

- Flexibility: the degree to which the recommendation permits interpretation and allows for alternatives in its execution

- Computability: the ease with which the recommendation can be operationalized in an electronic information system (only relevant if electronic implementation is planned) 


\section{Box 4: Essential elements of decision aids for patients}

- Evidence-based information on the condition, options and outcomes relevant to the patient's clinical situation

- Risk-based communication of the probabilities of potential outcomes and the levels of scientific uncertainty of each

- Clarification of values to ascertain which benefits, harms and scientific uncertainties are most important to the patient

- Structured guidance in the steps of deliberating and communicating with practitioners and significant others

minimize bias and improve the ability of patients to understand the probabilities of outcomes, evidence-based criteria are available for displaying probabilities within decision aids (Box 5). This is a crucial and complex step; we refer readers to the handbook by Jacobsen and O'Connor ${ }^{33}$ for specific details on how to frame the evidence. Third, the decision aid is reviewed by a panel of experts external to the development process. The panel may include clinicians, researchers, patients and others. Finally, the decision aid is evaluated by end-users. Defining a "good decision" is a challenge when no single "best" therapeutic action exists and choices depend on how patients value benefits versus harms. The International Patient Decision Aids Standards Collaboration has reached consensus on criteria for judging the quality of decisions (i.e., decisions should be informed and preference-based) and criteria for processes leading to high-quality decisions (i.e., recognize that a decision needs to be made, know options and their features, understand that values affect the decision, be clear about the options and features that matter most, discuss values with the clinician and become involved in preferred ways).

\section{Do decision aids work?}

A recent review of 10 systematic reviews of decision aids for patients showed that these knowledge tools improve patients' participation in decision-making, patients' knowledge of options, and agreement between patients' values and the subsequent treatment or screening decisions. ${ }^{34}$ Impact on clinical outcomes is less clear. ${ }^{34}$ When probabilities of outcomes are presented, patients have more realistic expectations of the chances of benefits and harms. The use of elective surgery (e.g., hysterectomy, prostatectomy, mastectomy, coronary bypass surgery, back surgery) decreased in favour of more conservative options without apparent adverse effects on health-related outcomes or anxiety. One trial evaluated the cost-effectiveness of patient decision aids for women experiencing benign abnormal uterine bleeding. ${ }^{35}$ Although no consistent effect on health status was observed, women who received information plus coaching to help them express their preferences had lower rates of hysterectomy compared with women in the control group (odds ratio [OR] 0.60; $95 \%$ confidence interval [CI] 0.38-0.96) and women who received information but no coaching (OR 0.52; 95\% CI 0.33-0.82]). Costs to the health system were lower for those who received education and coaching related to preferences.

A systematic review of 28 studies found that barriers to implementing decision aids for patients in clinical practice
Box 5: Criteria of IPDAS (International Patient Decision Aids Standards Collaboration) for the presentation of the probabilities of potential outcomes of different options $^{33}$

- Use event rates that specify the population and period

- Compare probabilities of potential outcomes using the same denominator, time period and scale

- Describe uncertainty of probabilities

- Use multiple methods to view probabilities (words, numbers, diagrams)

- Allow patients to view probabilities based on their own situation

- Place probabilities in context of other events

- Use both positive and negative frames

include practitioners' perceptions of the readiness of patients to use the aids, forgetting to offer them to patients, too complex or too simple content of thought of practitioners, time required to make them available, outdated evidence, cost and limited accessibility. ${ }^{36}$ The review also found that decision aids for patients are more likely to be used when they result in positive effects on patient outcomes or the clinical process, when patients prefer to actively participate in decision-making and when health professionals are motivated to use them.

\section{How do we determine the quality of decision aids?}

Although many decision aids for patients are available, they are, as with clinical practice guidelines, of variable quality. ${ }^{37}$ As a result, the International Patient Decision Aids Standards Collaboration was established to reach agreement on criteria for developing these decision aids and appraising their quality. ${ }^{32}$ The International Patient Decision Aids Standards checklist has domains that include essential content (i.e., providing information, presenting probabilities, clarifying values and guiding deliberation and communication), development (systematic process of development, balance, evidence base, plain language, disclosure) and evaluation (quality of decisions). ${ }^{32,38}$ This checklist is used to appraise decision aids available for patients in the Cochrane Decision Aid Inventory and the A-to-Z Inventory at a publicly available website, (www.ohri.ca/decisionaid). Validity and reliability testing of an International Patient Decision Aids Standards instrument is underway.

\section{Evidence-related gaps}

Research should focus on a number of areas to advance our understanding of knowledge tools. These areas include strategies to enhance understanding and implementation of systematic reviews (syntheses) and knowledge products such as guidelines and decision aids for patients. More research is needed to understand the impact of decision aids for patients on important clinical outcomes. Studies of the validity and reliability of the International Patient Decision Aids Standard are necessary. 
This article has been peer reviewed.

Competing interests: Dawn Stacey has been a consultant to Ortho Biotech Canada in a study of the decision-making needs of Canadians considering options for treatment of psoriasis. Annette O'Connor is the recipient of a research grant from the not-for-profit Foundation for Informed Medical Decision Making, which has a commercial link to Health Dialog, a company that promotes the use of decision aids for patients. None declared for Melissa Brouwers.

Contributors: All of the authors contributed to the conception and development of the article. Melissa Brouwers and Dawn Stacey drafted the manuscript. All of the authors critically revised the article for important intellectual content and approved the final version submitted for publishing.

Acknowledgement: We thank Sharon Straus for contributing the introductory portion of this article.

\section{REFERENCES}

1. Graham ID, Logan J, Harrison MB, et al. Lost in knowledge translation: time for a map? J Contin Educ Health Prof 2006;26:13-24.

2. Grimshaw JM, Santesso N, Cumpston M, et al. Knowledge for knowledge translation: the role of the Cochrane Collaboration. J Contin Educ Health Prof 2006;26: $55-62$.

3. Ioannidis JPA. Integration of evidence from multiple meta-analyses: a primer on umbrella reviews, treatment networks and multiple treatments meta-analyses. CMAJ 2009 DOI:10.1503/cmaj.081086.

4. Clinical Evidence. A guide to the text. How much do we know? London (UK): BMJ Publishing Group; 2009. Available: http://clinicalevidence.bmj.com/ceweb /about/knowledge.jsp (accessed 2009 Aug. 10).

5. Committee to Advise the Public Health Service on Clinical Practice Guidelines Clinical practice guidelines: directions for a new program. Washington (DC): National Academy Press; 1990.

6. National Guidelines Clearinghouse (NGC). Rockville (MD): Agency for Healthcare Research and Quality; 1998. Available: www.guideline.gov (accessed 2009 Sept. 10).

7. Browman GP, Snider A, Ellis P. Negotiating for change. The healthcare manage as catalyst for evidence-based practice: changing the healthcare environment and sharing experience. Healthc Pap 2003;3:10-22.

8. Palda VA, Davis D, Goldman J. A guide to the Canadian Medical Association handbook on clinical practice guidelines. CMAJ 2007;177:1221-6.

9. Program in Evidence-based Care. Program in evidence-based care handbook 2008. Toronto (ON): Cancer Care Ontario; 2008. Available: www.cancercare.on.ca/common /pages/UserFile.aspx?fileId=50876 $($ accessed 2009 Sept. 10$)$.

10. The guidelines manual 2007. London (UK): National Institute for Health and Clinical Excellence; 2007 Available: www.nice.org.uk/aboutnice/howwework /developingniceclinicalguidelines/clinicalguidelinedevelopmentmethods/theguidelines manual2007/the guidelines manual 2007.jsp (accessed 2009 Aug. 10).

11. Scottish Intercollegiate Guidelines Network. SIGN 50: A guideline developer's handbook. Edinburgh (UK): Scottish Intercollegiate Guidelines Network; 2008 Available: www.sign.ac.uk/guidelines/fulltext/50/index.html (accessed 2009 Aug. 10).

12. AGREE Collaboration Writing Group. Development and validation of an international appraisal instrument for assessing the quality of clinical practice guidelines: the AGREE project. Qual Saf Health Care 2003;12:18-21.

13. AGREE Research Trust [homepage]. The Trust; 2008 Available: www.agreetrust org (accessed 2009 Aug. 10).

14. Fretheim A, Schunemann HJ, Oxman AD. Improving the use of research evidence in guideline development: 3 . Group composition and consultation process. Health Res Policy Syst 2006;4:15.

15. Straus SE, Richardson WS, Glasziou P, et al. Evidence-based medicine: how to practice and teach it. Edinburgh (UK): Elsevier; 2005.

16. Atkins D, Eccles M, Flottorp S, et al. Systems for grading the quality of evidence and the strength of recommendations 1: critical appraisal of existing approaches. The GRADE Working Group. BMC Health Serv Res 2004;4:38.

17. Brouwers MC, Somerfield MR, Browman GP. A for effort: learning from the application of the GRADE approach to cancer guideline development. J Clin Oncol 2008;26:1025-6.

18. Atkins D, Briss PA, Eccles M, et al. Systems for grading the quality of evidence and the strength of recommendations 2: pilot study of a new system. BMC Health Serv Res 2005;5:25.
19. Guyatt GH, Oxman A, Vist G, et al. GRADE: an emerging consensus on rating quality of evidence and strength of recommendations. BMJ 2008;336:924-6.

20. ADAPTE Group. ADAPTE manual for guideline adaptation. The Group; 2007. Available: www.adapte.org (accessed 2009 Aug. 10).

21. Harrison M, Legare F. Adapting clinical practice guidelines to local context and assessing barriers to their use. CMAJ. DOI:10.1503/cmaj.081232.

22. Grimshaw J, Eccles M, Thomas R, et al. Toward evidence-based quality improvement Evidence [and its limitations] of the effectiveness of guideline dissemination and implementation strategies 1966-1998. J Gen Intern Med 2006;21(Suppl 2):S14-20.

23. Grimshaw JM, Thomas RE, MacLennan G, et al. Effectiveness and efficiency of guideline dissemination and implementation strategies. Health Technol Assess 2004;8:iii-iv, 1-72.

24. Grol R. Successes and failures in the implementation of evidence-based guidelines for clinical practice. Med Care 2001;39(Suppl 2):II46-54

25. Grol R, Dalhuijsen J, Thomas S, et al. Attributes of clinical guidelines that influence use of guidelines in general practice: observational study. BMJ 1998;317:858-61.

26. Grol R, Buchan H. Clinical guidelines: What can we do to increase their use? Med J Aust 2006;185:301-2.

27. Graham ID, Beardall S, Carter AO, et al. What is the quality of drug therapy clinical practice guidelines in Canada? CMAJ 2001;165:157-63.

28. Schiffman RN, Dixon J, Brandt C, et al. The guideline implementability appraisal [GLIA]: development of an instrument to identify obstacles to guideline implementation. BMC Med Inform Decis Mak 2005;5:23.

29. Shiffman RN, Dixon J, Brandt C, et al. Guideline Implementability Appraisal (GLIA) v1.0 [Internet]. New Haven (CT): Yale University; 2005. Available: http:/ /nutmeg.med.yale.edu/glia (accessed 2009 Sept. 10).

30. Manuel DG, Kwong K, Tanuseputro P, et al. Effectiveness and efficiency of different guidelines on statin treatment for preventing deaths from coronary heart disease: modelling study. BMJ 2006;332:1419.

31. O'Connor AM, Rostom A, Fiset V, et al. Decision aids for patients facing health treat ment or screening decisions: a Cochrane systematic review. BMJ 1999;319:731-4.

32. Elwyn G, O'Connor A, Stacey D, et al. Developing a quality criteria framework for patient decision aids: online international Delphi consensus process. BMJ 2006; 333:417.

33. Jacobsen MJ, O'Connor A. Population needs assessment: A workbook for assessing patients' and practitioners' decision making needs. Ottawa (ON): Ottawa Hospital Research Institute; 2006. Available http://decisionaid.ohri.ca/docs/implement /Population_Needs.pdf (accessed 2009 Oct. 9).

34. Coulter A, Ellins J. Effectiveness of strategies for informing, educating, and involving patients. BMJ 2007;335:24-7.

35. Kennedy AD, Sculpher MJ, Coulter A, et al. Effects of decision aids for menorrhagia on treatment choices, health outcomes, and costs: a randomized controlled trial. JAMA 2002;288:2701-8.

36. O'Connor AM, Stacey D, Entwistle V, et al. Decision aids for people facing health treatment or screening decisions [review]. Cochrane Database Syst Rev 2003;(2):CD001431

37. Sepucha KR, Fowler FJ Jr, Mulley AG Jr. Policy support for patient-centered care: the need for measurable improvements in decision quality. Health Aff (Millwood) 2004:(Suppl Web Exclusives):VAR54-62.

38. Gravel K, Legare F, Graham ID. Barriers and facilitators to implementing shared decision-making in clinical practice: a systematic review of health professionals' perceptions. Implement Sci 2006;1:16.

Correspondence to: Dr. Melissa Brouwers, Juravinski Cancer Centre, 699 Concession St., 4th floor, Rm. 206A, Hamilton ON L8V 5C2; fax 905 575-6308; mbrouwer@mcmaster.ca

The book Knowledge Translation in Health Care: Moving from Evidence to Practice, edited by Sharon Straus, Jacqueline Tetroe and Ian D. Graham and published by Wiley-Blackwell in 2009, includes the topics addressed in this series.

\section{Articles to date in this series}

- Straus SE, Tetroe J, Graham I. Defining knowledge translation. CMAJ 2009;181:165-8. 